
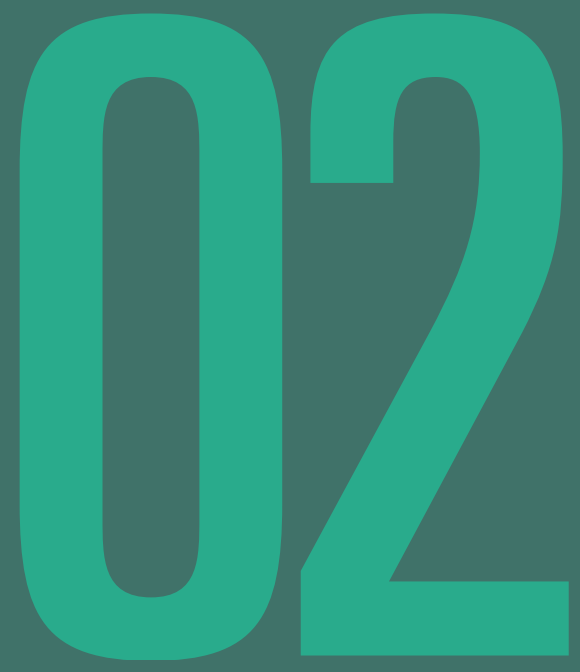

\title{
LA PLANEACIÓN TERRITORIAL A TRAVÉS DE LOS SISTEMAS COMPLEJOS EN UNA ERA DE CAMBIO AMBIENTAL. CASO: TURISMO
}

\section{Territorial planning through complex systems in an era of environmental change. Case: tourism.}

\author{
Leonardo Ayala Rodríguez \\ Leonardoayalar@uas.edu.mx \\ Universidad Autónoma de Sinaloa \\ Yazmín Paola Íniguez Ayón \\ arq.yazminpaola@gmail.com \\ Horacio Roldán López \\ trescascabeles@hotmail.com
}


Palabras clave: Sistemas complejos, turismo, cambio climático, territorio.

Keyword: Complex system, tourism, climate change, territory.

Resumen:

El presente artículo habla sobre la aplicación de los sistemas complejos como un instrumento de análisis en la actividad turística. En el primer apartado se analizaron los conceptos y paradigmas del cambio climático en relación con el turismo. En el año 2020, el turismo fue uno de los sectores económicos más afectados por la restricción sanitaria (COVID-19), lo cual trajo consigo una situación aún más complicada por los innumerables embates de fenómenos hidrometeorológicos extremos. La metodología propuesta se refiere a una exhaustiva revisión de los elementos que conforman el proceso de hacer turismo, así como a la problemática que enfrenta en la etapa de ordenación y planeación del territorio. El producto de nuestra investigación fue proponer un modelo de análisis para las diferentes variantes del turismo que juegan un papel muy importante en los procesos de configuración de la ciudad y el territorio. Los resultados de esta investigación pueden aplicarse en los procesos de planeación en el paradigma del desarrollo sustentable
Abstract:

This article talks about the application of complex systems as an instrument of analysis in tourism activity. In the first section, the concepts and paradigms of climate change in relation to tourism were analyzed. In 2020, tourism was one of the economic sectors most affected by the health restriction (COVID-19). Which brought with it an even more complicated situation due to the attacks of innumerable extreme hydro-meteorological phenomena. The proposed methodology refers to an exhaustive review of the elements that make up the process of tourism, as well as the problems faced in the processes of spatial planning and planning. The product of our research was to propose an analysis model for the different variants of tourism, which play a very important role in the configuration processes of the city and the territory. The results of this research can be applied in planning processes in the sustainable development paradigm 
Leonardo Ayala Rodríguez

Yazmín Paola Î́niguez Ayón

Horacio Roldán López

\section{INTRODUCCIÓN}

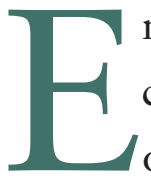

n México, como en la mayoría de las ciudades latinoamericanas, el crecimiento urbano es acelerado y en algunos casos con muy poca o nula planeación. La Nueva Agenda Urbana, específicamente en el punto 2, nos indica lo siguiente "Las poblaciones, las actividades económicas, las interacciones sociales y culturales, así como las repercusiones ambientales y humanitarias, se concentran cada vez más en las ciudades, y ello plantea enormes problemas de sostenibilidad en materia de vivienda, infraestructura, servicios básicos, seguridad alimentaria, salud, educación, empleos decentes, seguridad y recursos naturales, entre otros" (NAU, 2017, p.3).

La Ley General del Equilibrio Ecológico y la Protección al Ambiente (LGEEPA) (2010) es el máximo instrumento de política ambiental en México. La LGEEPA establece diferentes reglamentos y normas con el fin de administrar los recursos naturales ( $\mathrm{RN}$ ) de una manera eficiente bajo los criterios del desarrollo sustentable. Actualmente uno de los retos que enfrentan los países es la demanda y uso del suelo, que es el indicador básico de la planeación territorial en todos sus niveles. 
El Reglamento en Materia de Ordenamiento Ecológico (OE) es el instrumento técnico normativo para el proceso de ordenamiento ecológico en México. La planeación tiene que estar enfocada, por una parte, a evaluar y programar el uso óptimo del suelo y manejo de los recursos naturales. Por otra parte, pretende regular e inducir el uso más racional del territorio y el desarrollo de las actividades productivas. Bajo dichas premisas, tiene como fin proteger los ecosistemas y elaborar criterios de protección, conservación, restauración y aprovechamiento integral de los recursos, todo ello en el marco de desarrollo sustentable. El proceso de evaluación del uso del suelo en el territorio es un trabajo multidisciplinario que se puede abordar desde muchos flancos o disciplinas científicas como: la biología, ingeniería, química, urbanismo, geodesia y topografía, derecho, economía, ciencias sociales entre otras.

Los problemas de planeación radican en "ubicar" las actividades económicas de un sitio determinado, es decir que los usos del suelo configuran la estructura y funcionamiento de ciudades, regiones e incluso países. Ahora bien, si las ciudades funcionan a través de la geolocalización de sus actividades productivas y sus asentamientos humanos, es necesario considerar las estructuras o elementos naturales que forman, o deberían formar, pieza fundamental para los criterios de planeación.

La ecología entonces emerge como un elemento preponderante a considerar en la planeación de acuerdo con lo propuesto por Ludwig Von Bertalanffy, biólogo de origen austriaco que propuso la teoría general de sistemas (1976) y llegó a ser incluido en todas las áreas de la ciencia. En pleno siglo XXI, el término "sistema" ha embonado a la perfección para tratar de comprender las actividades económicas y las grandes crisis ambientales que vivimos. Lo anterior puede llevar a suponer que la complejidad no solo atañe al sistema, sino también al modelo que lo estudia; es decir, los modelos empleados para el estudio de los sistemas en el turismo también se han permeado de dicha complejidad (Palma, Franco, López y Velásquez, 2019). Es cierto que desde hace siglos se estudian los sistemas; sin embargo, la novedad en dicho concepto es 
la tendencia a estudiar los elementos que los conforman. De acuerdo con esta perspectiva, no es recomendable aislar los problemas o funciones del objeto de estudio.

La ecología profunda "No separa a los humanos ni a ninguna otra cosa del entorno natural. Ve el mundo, no como una colección de objetos aislados, sino como una red de fenómenos fundamentalmente interconectados e interdependientes" (Capra, 1996, p.29). Es decir, los procesos que contextualizan un fenómeno en particular son los responsables de guiar el proceso y encaminar hacia una solución del problema central.

La complejidad entonces trata de explicar el concepto de lo real en su forma tangible. Por otra parte, nos dice que el ambiente emerge como algo no pensado de la ciencia, así como también del conocimiento que ha negado la naturaleza que se manifiesta como una crisis ambiental y se vislumbra como la contraparte de la racionalidad problematizando las ciencias. El concepto de sustentabilidad parte del reconocimiento de la función que cumple la naturaleza como soporte, condición y potencial del proceso de producción (Leff, 2006).

El manejo a través de la interdisciplinaridad se guía por una visión global, un paradigma sistémico el pensamiento complejo. Bachelard, Canguilhem y Foucault consideran la interdisciplinariedad como método de reintegración al campo del conocimiento ambiental, fundado en la ecología (Leff, 2006). Por otra parte, se concluye que las catástrofes se encuentran cimentadas en las estructuras socioeconómicas practicadas durante largos periodos y como resultado no se puede culpar a un único factor (García, 2006).

Los fenómenos de la naturaleza, por ende, se consideran complejos debido a la interacción e interrelación de unos con otros, los cuales generan nuevos niveles de realidad al conocer dicha complejidad por un método que considere el "todo" y todas sus partes y la suma de ellas. Esta condición está implícita en todos los fenómenos climáticos y meteorológicos en una escala de tiempo mayor en los procesos coevolutivos y catastróficos de los cambios climáticos en nuestro planeta (Ritter, et al., 2002). 


\section{EL CAMBIO CLIMÁTICO COMO PRECURSOR DE UN CAM- BIO AMBIENTAL.}

En la primera conferencia mundial sobre el clima en 1979, se emitió una declaración en la que se involucraba a los gobiernos a prevenir y estimar los peligros potenciales del cambio climático. Se estableció un programa mundial sobre el clima a cargo de la Organización Meteorológica Mundial (OMM), el Programa de las Naciones Unidas para el Medio Ambiente (PNUMA) y el Consejo Internacional de Uniones Científicas (CIUC). Actualmente alrededor del mundo se están presentando diversos fenómenos derivados del incremento de la temperatura, como una deficiencia en servicios ambientales y un claro efecto negativo en el funcionamiento de las actividades antropogénicas.

En 1990, el Panel Intergubernamental del Cambio Climático (IPCC) publicó el primer Informe de evaluación sobre el estado del clima global, que tuvo impactos considerables entre los responsables de las políticas, tomadores de decisiones y responsables de la planeación. Puesto que los efectos son evidencia de un proceso de cambio ambiental, esto trae consigo una serie de efectos adversos en las actividades económicas, sociales y ambientales sin precedentes. El clima confiere características particulares a una porción territorial, aunando en primera instancia al identificar los riesgos potenciales del sitio; de tal suerte que el cambio en los patrones climáticos o efectos de un cambio climático es considerado como un sistema complejo y abierto, de acuerdo con la tipificación de García (2006). El estudio no se restringe a fronteras biológicas, físicas, sociales, económicas o políticas; de ahí el proceso de los sistemas complejos que analiza el estudio de cada uno de sus componentes y factores que influyen en cada proceso identificado.

Los gobiernos, en su afán de disminuir los índices de pobreza, optan por diferentes estrategias para mejorar sus economías. En México, se mantiene como objetivo prioritario erradicar la pobreza y le están apostando al sector turismo como eje transversal para mejorar dicha situación. Los principales efectos de eventos atípicos relacionados con el clima afectan las condiciones naturales de un lugar y, por consiguiente, propician condiciones no aptas para los residentes de un sitio, así como para los turistas. 


\section{EL TURISMO COMO PIEZA FUNDAMENTAL DEL DESARRO- LLO EN EL MARCO DEL CAMBIO CLIMÁTICO.}

El término "cambio climático", según el Panel Intergubernamental del Cambio Climático (IPCC, 2001), se considera una importante variación estadística en el estado medio del clima o en su variabilidad, que persiste durante un período prolongado. El cambio climático se puede deber a procesos naturales internos o a cambios del forzamiento externo, o bien a cambios persistentes en la composición de la atmósfera o en el uso de las tierras. La Convención Marco de Las Naciones Unidas sobre el Cambio Climático (CMCC), en su Artículo1, define "cambio climático" como: "Un cambio de clima atribuido directa o indirectamente a la actividad humana que altera la composición de la atmósfera mundial y que se suma a la variabilidad natural del clima observada durante periodos de tiempo comparables". La CMCC distingue entre "cambio climático" atribuido a actividades humanas que alteran la composición atmosférica y "variabilidad climática" atribuida a causas naturales.

El proceso de gestión integral del turismo se puede considerar a partir la siguiente premisa: "El producto turístico abarca una serie de elementos necesarios para la atención de las expectativas de consumo de los visitantes (atractivos, accesibilidad, equipamientos y servicios) al apropiarse de los territorios, la actividad turística se convierte en uno de los principales agentes intervinientes de la dinámica de las relaciones sociales previamente establecidas, engendrando un proceso dialéctico de producción de nuevas territorialidades" (Carvalho y Moquete, 2011, p.442). Por su parte, el Instituto Nacional de Ecología (INE) asocia el cambio de uso del suelo con la aptitud territorial que la define como: "la caracterización de la oferta ambiental en términos de capacidad productiva, la diversidad de ecosistemas que allí se disponen y los diferentes riesgos a los que están sujetos estos territorios”.

El proceso de globalización forzó a la actividad turística hacia una restructuración derivada de la competencia entre los distintos destinos alrededor del mundo. He ahí cuando un proceso de mercado incita a una actividad a 
reconfigurarse y emerger en el plano nacional e internacional como una de las actividades económicas mejor remuneradas, tan solo por debajo de la industria petrolera en el caso de México.

El turismo es de las principales actividades económicas en nuestro tiempo; la OMT lo define como un fenómeno social, cultural y económico. Está ínfimamente relacionado con el movimiento de personas a lugares que se encuentran fuera de su lugar de residencia habitual, ya sea por motivos personales o de negocios-profesionales. El turismo ha sido una de las pocas industrias que ha crecido considerablemente en tan poco tiempo. A partir de que los gobiernos se dieron cuenta del potencial económico, pusieron toda su atención en el turismo y vislumbraron en este una forma de combatir las grandes crisis mundiales (Jafar, 2005).

Fennell y Plummer publicaron en el 2009 uno de los primeros artículos que propone el uso de nuevos enfoques de gestión para lograr la sustentabilidad del turismo. Con ello, buscaron lograr la sustentabilidad a través del enfoque en aspectos como los conflictos entre múltiples grupos de interés, la complejidad sistémica y la incertidumbre como temas importantes que requieren respuestas por parte de los responsables de la gestión turística (Citado en Oyarzun et al., 2018). El turismo tuvo que adecuarse a los nuevos desafíos con su fórmula mejorada el “Turismo Sostenible". La OMT reconoce los efectos generados en un proceso de cambio que se traduce en: golpes de calor, enfermedades transmitidas por vectores (enfermedades zoonóticas), escasez de agua y alimentos. Los impactos del clima repercuten en el estado de confort y en la salud de las personas, tanto como en la seguridad de un sitio; son factores determinantes en la selección de un lugar (Declaración de Djerba, 2003).

La OMT ha estimado que el primer trimestre del 2020 la actividad turística se desplomó en un 22\%, producto de la pandemia COVID-19 (OMT, 2020). La preocupación es tal, puesto que la captación de divisas por este rubro iba a la alza; tan solo para México, el turismo reportó ingresos por 21,333 millones de dólares, según la Secretaría de Turismo para el año 2018 (SECTUR, 2018). Esto sitúa a la actividad turística como una fuente confiable de 
obtención de divisas para nuestro país; la planeación y el proceso de evaluación en los sitios donde se desarrolla esta actividad. Si está bien organizado, puede ser un complemento para el desarrollo de las comunidades, para el rescate de su propia identidad y para los recursos naturales en algún estatus de riesgo (Palmas et al., 2019).

En nuestro país una proporción muy importante de la población depende y se localiza en la zona costera, que por sus atributos ambientales alberga algunas de las actividades más importantes tales como: la agricultura, la acuacultura y el turismo, para el caso del noroeste de México. La zona costera se enfrenta a múltiples retos; desde la contaminación de sus aguas, hasta la destrucción y disminución de sus servicios ambientales y problemas entre diferentes sectores por el espacio físico y la temporalidad (Rivera, et al., 2020).

La OMT reconoce la importancia de los servicios ambientales en la selección de destinos. El cambio climático aparece entonces como una actividad ligada en el proceso de hacer turismo en el ámbito internacional y nacional; como una forma de cuantificar los embates, efectos y adaptación. En las últimas décadas, los cambios en los patrones del clima han tenido un impacto en los sistemas naturales y antrópicos alrededor del mundo. Estos impactos reflejan una clara sensibilidad de los sistemas naturales y humanos al clima cambiante (IPCC, 2014).

Los pronósticos del IPCC no son nada alentadores para el turismo mundial y no consideraban en sus proyecciones, por lo menos de manera particular, una pandemia mundial en tan poco tiempo; ya que una de las conclusiones del Panel Intergubernamental de Cambio Climático (IPCC, 2007) es que hay una alta probabilidad de que haya un aumento en olas de calor, tormentas intensas, temperaturas mínimas más altas con menos días fríos. También es probable que aumente la intensidad del viento máximo y haya una mayor presencia de ciclones tropicales de categorías medias y altas. El aumento en el nivel del mar afectará a la industria hotelera asentada en esta zona ya que se tendrían efectos más severos de las mareas de tormenta con afectaciones a la zona costera 
y la arena de la playa. Se presentaría una competencia por el recurso hídrico entre las zonas urbanas (población) y las zonas hoteleras (turistas) ante una disminución en la disponibilidad del recurso. Todo lo anterior se enmarca en una crisis de salud sin precedentes que la OMT identifica como un nuevo paradigma y define como la capacidad excepcional del turismo para crear oportunidades fuera de las grandes ciudades y preservar en todo el mundo el patrimonio cultural y natural (OMT, 2017).

\section{GENERANDO UN MODELO DE ANÁLISIS}

Los modelos clásicos de ordenación y planeación de alguna forma consideran los criterios básicos de la sustentabilidad. Sin embargo, la teoría de sistemas viene a cambiar entonces la concepción de los objetos de interés. En contraparte, nos invita a abrir aún más el panorama, así como las interacciones para ser examinadas en un mismo periodo. Dicho lo anterior, el concepto de sistema muta para encajar en la descripción y solución de los problemas ambientales, el desarrollo urbano y territorial y cualquier fenómeno de cualquier disciplina.

Los sistemas complejos nos enmarcan los niveles de análisis de los fenómenos, donde los procesos de primer nivel son esencialmente locales; los procesos de segundo nivel son regionales o nacionales; los de tercer nivel son internacionales. Todos ellos tienen formas distintas de desarrollarse. Los niveles de análisis de mayor escala explican los de menor escala y viceversa. Sin embargo, la tendencia de explicar los fenómenos naturales o las crisis globales responde más atinadamente a los sistemas complejos.

En el año 2001, el IPCC publica los conceptos para entender el fenómeno del cambio climático. En un primer acercamiento se reconoce como responsable que da lugar a dicha condición a los gases de efecto invernadero GEI'S, considerados los responsables directos de la regulación de la temperatura en la tierra. El cambio climático se puede deber o no a la influencia del hombre con la quema de combustibles fósiles; sin embargo, las repercusiones engloban muchos elementos a considerar. Los sectores económicos sufren o parecen sufrir 
los estragos del C.C., y han tenido que irse adaptando a los paradigmas como la sustentabilidad. Todas las actividades primarias y de servicios han tenido que coevoluvionar con los cambios en los patrones del clima regional.

El turismo no puede ser ajeno a los problemas ambientales, por lo que hace lo propio y de nuevo muta para contextualizar su existencia en el marco del cambio climático. Para ello se propuso como método de análisis el siguiente diagrama que engloba los principales elementos que tendríamos que considerar en un momento determinado para realizar la planificación y regulación de los nuevos destinos. Lo anterior se enmarca en el concepto de turistificación que se define como el proceso por el cual se transforman las condiciones ambientales, históricas, sociales o culturales de un sistema (que se puede tipificar como territorio) en un producto valioso en el mercado turístico (Knafou, 1999) Figura 1.

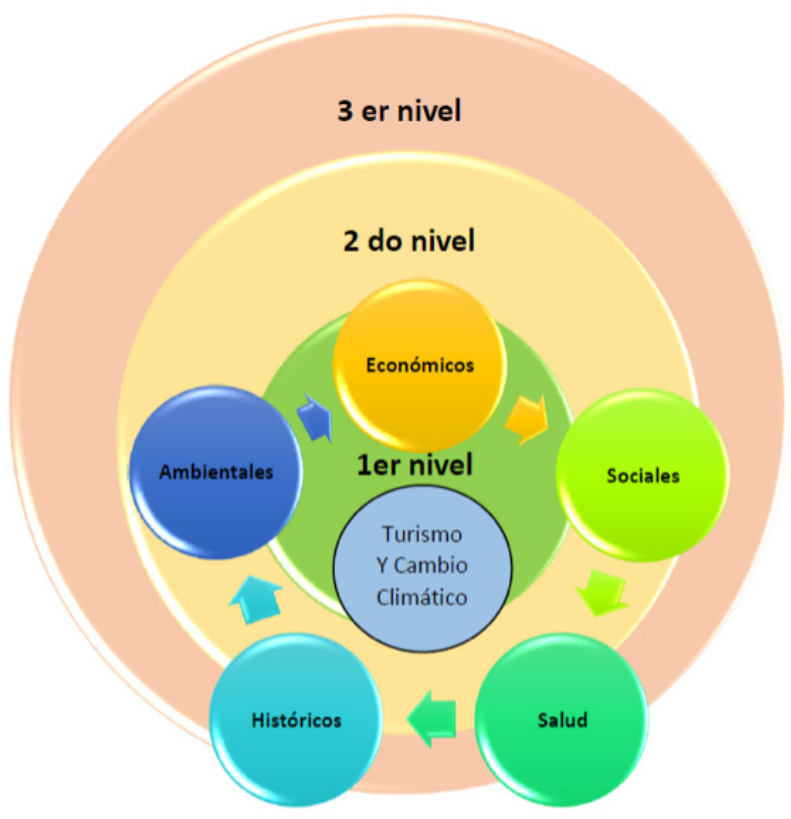

Figura 1.-Esquema de análisis de acuerdo con los sistemas complejos para el proceso de la actividad turística en el marco del cambio Climático. Elaboración Propia de acuerdo con García, 2006.

\section{ER NIVEL: ENTORNO DEL PROBLEMA}

De acuerdo con el planteamiento del problema se identificaron los componentes del sistema complejo constituyendo los subsistemas siguientes: Ambiental, Social, Económico. 


\section{SUBSISTEMA AMBIENTAL}

Se trata del funcionamiento sistémico de cuencas, vegetación (distribución de la vegetación, tipo y condiciones de la vegetación, deforestación), factores climáticos (precipitación, temperatura, humedad, etc.) incendios, erosión, incidencia de eventos hidrometeorológicos, estatus de la fauna (distribución), usos de suelo, aptitud, vocación y cambio de uso de suelo. Los elementos anteriores nos ayudan a determinar el riesgo de ocurrencia derivado de la exposición y vulnerabilidad de la zona a evaluar. Los resultados obtenidos se pueden representar en unidades de paisaje, unidades ambientales, zonas ecológicas o la que corresponda a la escala y el instrumento seleccionado.

\section{SUBSISTEMA SOCIAL}

Los indicadores socioeconómicos incluyen la distribución de las unidades Económicas (productivas) tomando como base el Directorio Estadístico Nacional de Unidades Económicas (DENUE), integrado con información de identificación y ubicación de las unidades económicas captadas por los censos económicos del 2019. En lo referente a la evolución de la población ocupada para cada municipio y sus Unidades Ambientales, se consideran los datos del estado de Sinaloa para los años 2000 y 2020. La visión competitiva de la economía constituye un instrumento metodológico idóneo para diseñar las políticas de desarrollo económico, al contemplar factores que potencian la capacidad del territorio de atraer y retener inversiones para detonar procesos económicos a mediano y largo plazo. Así, el desarrollo de la economía está íntimamente ligado con la condición de los recursos hídricos, la energía y la adaptación de las actividades productivas en un clima cambiante; acceso a servicios, recursos hídricos, vulnerabilidad ante fenómenos climáticos extremos, salud, y alimentación.

\section{SUBSISTEMA ECONÓMICO}

El clima global es un bien público y, por tanto, el cambio climático desde una óptica económica representa la mayor externalidad negativa global y, dada su magnitud, determinará en gran medida las características y condiciones del desarrollo económico en este siglo. Es a partir de la identificación del problema y sus componentes o subsistemas que surge la búsqueda de una solución o una explicación en el marco de los lineamientos nacionales e internacionales. 


\section{SUBSISTEMA SALUD}

Este tópico sin duda es el que actualmente tiene más relevancia ante la falta de planes de contingencia a tan gran escala. Para este subsistema aún no hay mecanismos ni proyecciones tangibles hasta el momento; sin embargo, se puede hablar sobre las estrategias emergentes como en los casos de pandemias como la que ocurre en estos momentos.

\section{DO NIVEL: ENTORNO NACIONAL: CASO MÉXICO.}

Planes Nacionales de Desarrollo, Leyes Federales y planes Estatales y municipales. En el Diario Oficial de la Federación del 25 de abril del 2005, aparece el acuerdo por el que se crea con carácter permanente la Comisión Intersecretarial de Cambio Climático (CICC), por México en la Convención Marco de las Naciones sobre el Cambio Climático (CMNUCC) en la materia y demás instrumentos derivados de esta. La CICC está integrada por siete secretarías de estado. La CICC elaboró la Estrategia Nacional de Cambio Climático (ENACC, 2007). El tema de Cambio Climático ha sido incluido por primera vez en dicho plan en su eje rector 4 dedicado a la sustentabilidad ambiental.

\section{LEY GENERAL DEL CAMBIO CLIMÁTICO (LGCC)}

La ley general del cambio climático dice en el artículo 1 "La presente ley es de orden público, interés general y observancia en todo el territorio nacional y las zonas sobre las que la nación ejerce su soberanía y jurisdicción y establece disposiciones para enfrentar los efectos adversos del cambio climático" (Diario Oficial de la Federación, 2012).

\section{LEY GENERAL DE TURISMO (LGT)}

La materia turística comprende los procesos que se derivan de las actividades que realizan las personas durante sus viajes y estancias temporales en lugares distintos al de su entorno habitual, con fines de ocio y otros motivos. Los procesos que se generan por la materia turística son una actividad prioritaria nacional que, bajo el enfoque social y económico, genera desarrollo regional. (Diario Oficial de la Federación, 2009) 
Ley general de asentamientos humanos, ordenamiento territorial y desarrollo urbano.

Ordenamiento territorial de los asentamientos humanos: el ordenamiento territorial es una política pública que tiene como objeto la ocupación y utilización racional del territorio como base espacial de las estrategias de desarrollo socioeconómico y la preservación ambiental (Diario Oficial de la Federación, 2016).

\section{ER NIVEL: ENTORNO INTERNACIONAL}

Cumbre de Río: Agenda 21: Plan de acción que tiene como finalidad metas ambientales y de desarrollo en el siglo XXI. La Convención Marco de las Naciones Unidas sobre Cambio Climático (CMNUCC). El texto de la CMNUCC, signado por la mayoría de los países, fue firmado por México el 13 de junio de 1992 y ratificado el 11 de marzo de 1993. En este sentido, forman parte del Anexo1 los países industrializados que fueron miembros de la Organización para la Cooperación y Desarrollo Económicos (OCDE) en 1994.

\section{EL PROTOCOLO DE KYOTO}

El Protocolo de Kyoto de la citada Convención es una adhesión acordada por los países para adoptar medidas y establecer compromisos, en torno a lo ya establecido sobre cambio climático y las acciones para reducir el calentamiento atmosférico. El Protocolo de Kyoto entró en vigor el pasado 15 de febrero de 2005. La decimoctava Conferencia de las Partes (COP 18) sobre cambio climático ratificó el segundo periodo de vigencia del Protocolo de Kyoto desde el 1 de enero de 2013 hasta el 31 de diciembre de 2020.

\section{LA AGENDA 2030}

Se propone poner fin a la pobreza en todas sus formas en todo el mundo, poner fin al hambre, lograr la seguridad alimentaria y la mejora de la nutrición y promover la agricultura sostenible. Así mismo, pretende garantizar una vida sana y promover el bienestar para todos en todas las edades. 


\section{CONCLUSIONES}

Los problemas actuales como la crisis alimentaria, uso del agua, fenómenos climáticos extremos y de salud, derivados del aumento en la temperatura media de la tierra, se pueden detectar en los procesos de segundo y tercer nivel, enfatizando en los siguientes puntos: utilización abusiva del medio físico y, con ello, degradación (muchas veces irreversible) y marginación de sectores sociales que ven deteriorarse sus niveles de vida (García, 2006). "La diferencia entonces entre la noción de complejidad derivada de los primeros pensadores, hasta la referencia de los científicos contemporáneos, se puede considerar como un proceso de construcción y deconstrucción, sobre una investigación recolectando experiencias del trabajo mismo" (Serrano et al., 2012, p. 9). Las conclusiones del Panel Intergubernamental de Cambio Climático se refieren a una alta probabilidad de que haya un aumento en olas de calor, tormentas intensas, temperaturas mínimas y más altas con menos días fríos. La presencia de huracanes de categorías medias tendrá aun mayor incidencia en zonas vulnerables como la zona costera. La formación de ciclones tropicales se debe en gran medida al aumento de la temperatura medida del mar.

La actividad agropecuaria y el sector turismo han sido unas de las más afectadas en México, ya que la mayoría de ellas se realizan en localidades próximas a la costa, esto aunado a la falta de visitantes por la contingencia sanitaria. Los efectos negativos, como en la infraestructura de zonas costeras, han afectado numerosos destinos turísticos. El aumento en el nivel del mar afectará la industria hotelera asentada cerca del mar; se tendrían efectos más severos de las mareas de tormenta con afectaciones a la zona costera y playa. Tanto el cambio climático como el turismo han sido estudiados desde diferentes perspectivas y de forma paralela; sin embargo, a partir de los paradigmas de la sustentabilidad han tomado un camino en común.

En este sentido van dirigidas las evidencias de un cambio en los sistemas naturales y en los patrones regulares del clima. Es necesario aplicar mecanismos aún más específicos para la construcción de escenarios y toma de decisiones. Los sistemas complejos pueden sistematizar el proceso para generar indicadores claves para la implementación de obras, infraestructura y programas, con el fin 
de impulsar la adaptación y mitigación que tanto se busca. En este momento es preponderante la adaptación, ya que los efectos como sequias, inundaciones, plagas, enfermedades ya están presentes. Por otra parte, la mitigación tendría que jugar rol a mediano y largo plazo, por los largos periodos de residencia de los gases de efecto invernadero (GEI'S) en la atmosfera.

Los instrumentos de gestión ambiental para la planeación tipificados en algún nivel propuesto en este artículo nos ayudan a construir y fortalecer uno de los objetivos primordiales del marco jurídico ambiental en México; en aras de fortalecer la capacidad de resiliencia de los sistemas ambientales y por ende las actividades que se desarrollan en ellos. Ante tal situación, se deben tomar medidas de adaptación y mitigación para la construcción de los complejos turísticos; a pesar de que el Fondo Nacional del Turismo (FONATUR) seguía utilizando hasta hace unos años el mismo modelo de enclave turístico desde hace casi 40 años. Este generaba procesos de polarización espacial y social en los territorios donde se proyectaban los Centros Integralmente Planeados (CIP), ya en decadencia o extintos.

En el país, se prolonga por tiempo indefinido la falta de un modelo sustentable para el desarrollo de los centros turísticos, por lo que se están creando condiciones irreversibles de deterioro de los ecosistemas. La prevención es una estrategia fundamental para el desarrollo sostenible, dado que permite administrar los recursos naturales evitando el uso inadecuado de los servicios ambientales (Magaña, 2009).

Los sistemas complejos nos ayudan a reconfigurar y considerar la mayor cantidad de procesos: ambientales, económicos y sociales. Estos resultan fundamentales para el diagnóstico, análisis y soluciones de un problema o una actividad bajo los criterios del desarrollo sustentable. Así mismo, contribuye a realizar reformas en los instrumentos jurídicos y mecanismos de fondo para la ordenación del territorio y la planeación de ciudades en un mundo en y postpandemia. 


\section{REFERENCIAS}

Bertalanffy, L. V., (1976) Teoría General de los Sistemas. FCE, México.

Capra, F. (1996). La trama de la vida. Una nueva perspectiva de los sistemas vivos. Barcelona: Anagrama.

Carvalho, K. D. y Moquete-Guzmán, S. J. (2011). El Turismo en la Dinámica Territorial ¿Lógica global, desarrollo local? Estudios y Perspectivas en Turismo, 441-461.

Comisión Intersecretarial del Cambio Climático. (2007). Estrategia Nacional de Cambio Climático. México: SEMARNAT. Instituto Nacional de Ecología y Cambio Climático (INECC). (15 de octubre de 2020). Recuperado de: https:// cambioclimatico.gob.mx/mexico-ante-el-cambio-climatico-accion-climatica/

De Alba, E. (2004). "La Convención Marco de las Naciones Unidas sobre el Cambio Climático." Cambio climático: una visión desde México. México: INE.

Djerba Declaration. Climate Change and Tourism (2003). Recuperado de www. unwto.org

García, R. (2006). Sistemas complejos Conceptos, método y fundamentación Epistemológica de la investigación interdisciplinaria. Barcelona: Genisa.

Panel Intergubernamental del Cambio Climático. (2007). Grupo Intergubernamental de Expertos sobre Cambio Climático IPPC. Cambio climático 2007 Informe de Sintesis. Ginebra, Suiza: Grupo Intergubernamental de Expertos del Cambio Climático.

Panel Intergubernamental del Cambio Climático. (2014). Impactos observados, vulnerabilidad y adaptación en un mundo complejo y cambiante. Grupo de trabajo II DEL IPCC.

Jafari, J. (2005). La cientificación del turismo. Revista Contribuciones a la Economía. Recuperado en http://www.eumed.net/ce/2005/jafari.htm. 
Leff, E. (1998). Saber Ambiental: Sustentabilidad, Racionalidad, Complejidad y Poder. México: Siglo veintiuno.

Diario Oficial de la Federación. (2010). Ley General del Equilibrio Ecológico y la Protección al Ambiente. México, el 28 de enero del 1988.

Diario Oficial de la Federación. (2016). Ley General de Asentamientos Humanos, Ordenamiento Territorial y Desarrollo Urbano. México, el 28 de noviembre del 2016.

Diario Oficial de la Federación. (2018). Ley General de Turismo. México, el 17 de junio de 2009.

Diario Oficial de la Federación. (2012). Ley General del Cambio Climático. México, el 06 de junio de 2012.

Magaña, V., Pérez, J. L., Vázquez, J. L., Carrisoza, E., y Pérez, J. (1999). El Niño y el clima. Los impactos de El Niño en México, 23-66.

Naciones Unidas. (2000). Agenda 21. Naciones Unidas por el Desarrollo Sustentable. Recuperado de https://www.un.org/spanish/esa/sustdev/agenda21/ agenda21sptoc.htm

Naciones Unidas. (2017). Nueva Agenda Urbana. Quito: Secretaría de Habitat III.

Naciones Unidas. (2018). Agenda 2030 y los Objetivos del Desarrollo Sostenibble. Santiago: Naciones Unidas.

Organización Mundial del Turismo (2020). Covid 19 respuesta. Recuperado de https:// www.unwto.org/es/turismo-covid-19

Organización Mundial del Turismo OMT (2017). Cambio Climático y Turismo. Programa de las Naciones Unidas para el Medio Ambiente, Organización Meteorológica Mundial. Davos, Suiza. 
Oyarzun, L. y Taucare Taucare, H. (2018). El cambio de paradigma en el turismo sustentable. Las implicancias para su gestión. Estudios y Perspectivas del Turismo, 140-157.

Palmas-Castrejón, Y. D., Franco-Bravo, A. I., López-Zapata, L. V. y Giraldo-Velásquez, C. M. (2019). Sistemas complejos y turismo: aplicación del modelo de turismo armónico en dos localidades de países latinoamericanos. Cuadernos de Geografía: Revista Colombiana de Geografía, 29(2), 354-372.

Ritter, W., Guzmán, S., Sánchez-Santillán, N., Suarez, J., Corona, C., Muñoz, H., ... y Pérez, T. (2002). El clima como sistema complejo adaptativo en coevolución. Ciencia y Mar, 6(17).

Rivera-Arriaga, E., Azuz-Adeath, I.,Cervantes- Rosas, O. D., Espinoza-Tenorio, A. Silva Casarín, R. Ortega-Rubio, A. Botello, A. V. y Vega-Serratos, B. E. (2020). Gobernanza y Manejo de las Costas y Mares ante la Incertidumbre. Una Guía para Tomadores de Decisiones. Universidad Autónoma de Campeche: Ricomar.

Secretaría de turismo (2018). Visión Global del Turismo a México. México: Secretaría de Turismo. SECTUR.

Serrano-Barquín, R. D. C. S., Cruz- Jiménez, G. C., Zepeda, F. A., Osorio-García, M. O., y Sánchez-Barreto, R. F. S. (2012). La complejidad, expresión de nuestro tiempo: el turismo desde los sistemas complejos. CULTUR: Revista de Cultura e Turismo, 6(1), 4-24. 